\title{
On-Line Training of the Path-Loss Model in Bayesian WLAN Indoor Positioning
}

\author{
Luigi Bruno, Mohammed Khider and Patrick Robertson \\ Institute for Communications and Navigation \\ German Aerospace Center (DLR) \\ Wessling, Germany \\ Email: \{luigi.bruno,mohammed.khider,patrick.robertson\}@ dlr.de
}

\begin{abstract}
Received signal strengths have been widely used in indoor positioning due to the massive presence of wireless local networks in buildings. To avoid long training phases theoretical propagation models such as the path-loss model can be used. The main issue is that the path-loss parameters, namely the transmitted power and the decay exponent, can assume a wide range of values, depending on devices, building structure and other environmental features.

In this paper, we propose a Bayesian positioning algorithm based on the Rao-Blackwellized particle filter, where the system estimates the parameters of the path-loss model independently for each AP in addition to localizing the user. Both parameters are described by discrete variables and their probability distribution is estimated starting from a uniform prior. We validate the algorithm with simulations and two different experiments; finally, some remarks on complexity are also given.
\end{abstract}

\section{INTRODUCTION}

Reliable indoor localization techniques are required to provide Location Based Services in buildings and urban canyons, e.g., navigation in airports and malls and support of first-aid units [1], [2]. Satellite navigation systems are inaccurate indoors due to multipath, therefore techniques based on local and low cost sensors are needed [3]. Therefore, the sensors which are already available in a mobile phone, like accelerometers, compass, magnetometers, and radio receivers, are gathering a growing interest, both in scenarios where they are employed alone and when their measurements are fused, e.g. according to a probabilistic paradigm [4].

We focus on wireless local area networks (WLANs), which are increasingly spreading indoors. WLAN based localization makes use mainly of Received Signal Strengths (RSSs) of the beacon signals which are periodically sent by the Access Points (APs); it does not require any sensitive information exchange between user and network, in agreement with privacy issues [5].

Limits to RSS based localization are usually imposed by the characterization of the radio channel, that is still an open issue due to multipath and blockage yielded by the building structure, the materials of walls and furniture and the presence of metallic objects and people. Furthermore, it has been widely shown how propagation features can harshly change within short and long term intervals [6].

Fingerprinting techniques avoid radio propagation modeling and rather rely on extensive measurement campaigns across the area under test [7]. Hence, a radio map (RM) is built as a collection of RSS vectors associated to known positions and during the localization stage the vectors in the RM are compared to the upcoming RSS vectors: the user's position is then estimated based on a clustering algorithm. RADAR, presented in [7], is still today a popular fingerprinting algorithm: the RM contains only the average of the RSS vectors collected in each position and clustering is based on the minimization of the Euclidean distance. Fingerprinting achieves localization accuracy down to 2-3 meters if the RM covers sufficiently the area under test. The algorithm stability is, however, put in trouble by the environmental changes, that are usually approached by map corrections. To do so, [8] proposes the RM correction by means of a linear transformation under the arbitrary assumption that the change is uniform across the area, while the algorithm in [9] makes use of Model Trees to adapt the RM online without assuming explicit transformation functions. Both algorithms use measurements at reference nodes, and therefore additional hardware, in order to detect and evaluate the changes. More recently, [10] proposed a technique based on principal component analysis to extract features from the RM without reference nodes.

An alternative approach to WLAN based fingerprinting localization employs theoretical models and resorts to geometric principles, like trilateration, to localize the user [7], [11], [12]. The theoretical approach is usually not as accurate as fingerprinting, but it avoids any RM construction; furthermore, propagation models can be stated as functions of parameters, whose calibration is used to fit environmental features. We focus on the parameters calibration in the case of the pathloss model [7], [11]. To do so, we avoid both a training phase and the use of reference nodes by developing an adaptive algorithm based on Bayesian probabilistic theory, in which the parameters are learned while performing localization. The APs are deployed in known positions and we assume independent outcomes of the path-loss parameters for each AP.

In a previous paper a similar framework is proposed in which only one parameter of the path-loss model is estimated per AP, namely the transmitted power [13]. The parameter is stacked into the state vector and estimated by means of a particle filter together with the user's trajectory; the path-loss exponent, which describes the decay of power with distance, is instead not estimated but the algorithm is rather fed with 


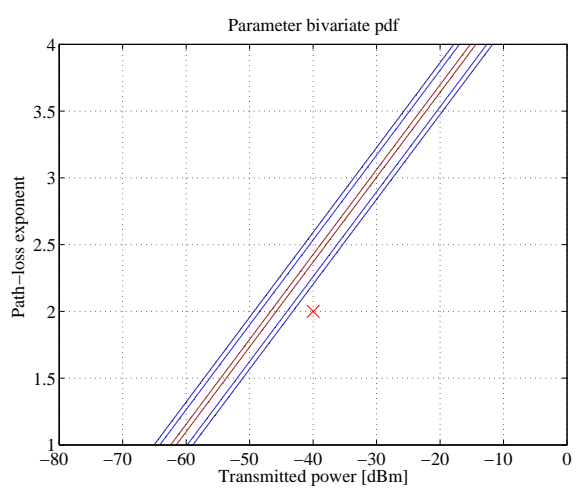

(a) $\mathrm{k}=1$

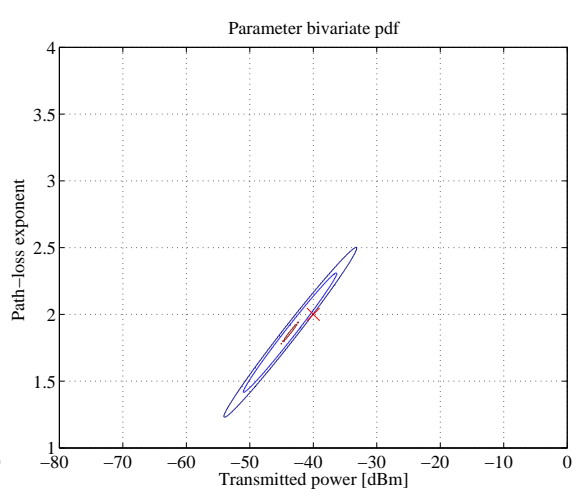

(b) $\mathrm{k}=5$

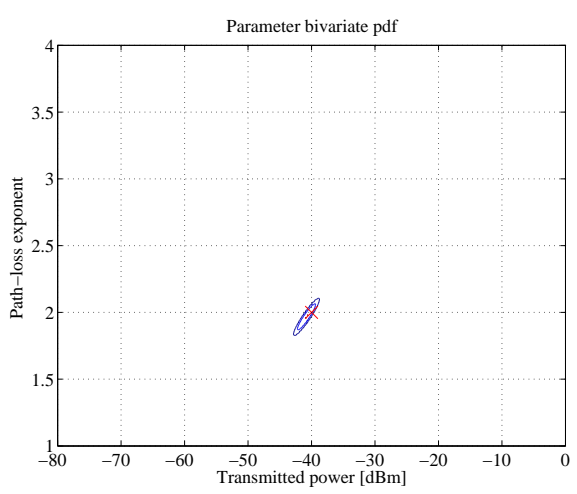

(c) $\mathrm{k}=20$

Fig. 1. RSS likelihood function conditioned on $r, h$ and $\alpha$; the plots are obtained by assuming perfect knowledge of $r$ and by computing the likelihood function on a range of values for the path-loss parameters. The likelihood function is computed with (a) $k=1$, (b) $k=5$ and (c) $k=20$ independent measurements. Distance $r$ is randomly generated from a pdf that is uniform between $d_{0}=1.6 \mathrm{~m}$ and $15 \mathrm{~m}$ and all outcomes are independent.

the free-space value, that is 2 . In real scenarios, however, the path-loss exponent is reported to vary in the range of 1 to 4 [11]. Although localization performance improves even by estimating only the transmitting power, relevant potential lies in the determination of the path-loss exponent, as shown in the literature both in an experimental scenario [14] and according to theoretical discussions [15]. Authors in [16] propose a RSSbased localization algorithm in which the path-loss exponent is considered unknown. Although they do not estimate it in an explicit way, they implicitly account for it. In fact, the range estimations which are yielded by the RSS are combined with a spring-relaxation method: each AP-user distance is modeled by a spring, whose elasticity coefficient is made variable with the distance in order to mitigate the path-loss exponent inaccuracy. This approach is based on the claim that such a mismatch yields an error in the distance determination that is proportional to the distance itself; therefore, they propose to increase the variance of weak RSS measurements to account for the error caused by the parameter mismatch. [17] adopts the joint estimation of both transmitted power and pathloss exponent by performing a training stage during which a maximum likelihood estimation of both parameters is made.

Differently from the other approaches, we propose an RSSbased localization algorithm in which the path-loss parameters are gradually evaluated for all APs together with the user's position. The algorithm is based on Bayesian probability and is implemented by means of a Rao-Blackwellized particle filter (RBPF) in which, for all particles, the user's position is sampled based on a predictive model and the RSS measurements are used to generate the particle weight. The parameters are modeled as independent discrete variables, with possible values in suitable sets. Each pair of values for the transmitted power and the path-loss exponent represents a hypothesis, whose probability is updated at every new measurement for all particles. Dealing with probability distributions rather than point-wise estimates of the parameters makes the algorithm more robust. This is shown by means of both computer and real world experiments. In the last case, the user's move- ment model is improved by employing inertial measurements from a foot-mounted Inertial Measurement Unit (IMU). Step measurements obtained by processing inertial measurements with a Zero Velocity Updates (ZUPT) filter are affected by a drift which causes a growing error in localization [18], [19]. RSS measurements from few APs can correct this error and produce very accurate results when the path-loss model is well calibrated, and this is done on-line without a training phase. The price paid is a transient phase during which the pathloss parameter distributions are gradually refined, but then the algorithm convergence yields a localization accuracy that is suitable till here to many indoor navigation applications.

The paper is structured as follows: Section II presents the path-loss model and a discussion on the parameter estimation; in Section III we develop the Bayesian filter and its implementation in terms of RBPF, whose complexity is also discussed; simulation and experimental results are instead discussed in Sections IV and V, respectively, while concluding remarks will be given in Section VI.

\section{PATH-LOSS MODEL AND PARAMETERS}

The path-loss model describes the value of the signal power which propagates over a finite distance and it is an extension of the Friis formula. According to it, the power $P(r)$ received at distance $r$ is given by [11]

$$
P(r)=P_{0}\left(\frac{d_{0}}{r}\right)^{\alpha},
$$

where $d_{0}$ is a reference distance, $P_{0}=P\left(d_{0}\right)$ is a constant power representing the transmitted power and antenna gains and $\alpha$ is the path-loss exponent ( $\alpha=2$ in free space). This model is valid in far field condition, as for $r \rightarrow 0, P(r) \rightarrow \infty$. In this paper, $d_{0}$ is the limit between near and far field, i.e., we assume $r \geq d_{0}$ and, therefore, $P(r) \leq P\left(d_{0}\right)$.

Restating (1) in $\mathrm{dBm}$ for the signal strength $h(r)$ (square root of power) we find:

$$
h(r)=h-20 \alpha \log _{10}\left(r / d_{0}\right),
$$


where $h=10 \log _{10} P_{0}$ is the transmitted power in $\mathrm{dBm}$. RSS measurements in $\mathrm{dBm}$ are corrupted by a Gaussian white noise with mean given by (2) and variance denoted by $\sigma_{y}^{2}>0$, i.e.:

$$
y \sim \mathcal{N}\left(h(r), \sigma_{y}^{2}\right) .
$$

The Gaussian probabilistic model is used in the literature in environments where slow fading is going to be relevant; it is shown that it corresponds for the signal strength in Watt to a Lognormal probability density function (pdf) [7], [11], [13].

The path-loss parameters are, in our case, the transmitted power $h$ and the exponent $\alpha$ in (2). We here consider them unknown but static, at least over a sufficiently short time interval (our experiments last few minutes). Their joint estimation, combined with inaccuracy on the user's position and with RSS noise, could be prevented by ambiguity. As an example, we refer to simulation scenarios where $k$ independent measurements are sampled according to the model (3), with $\sigma_{y}=3 \mathrm{dBm}$ and $r$ randomly varying between $d_{0}=1.6 \mathrm{~m}$ and $15 \mathrm{~m}$. In Fig. 1 we show the likelihood function of the measurements conditioned on the distance $r$ and on the parameters $h$ and $\alpha$; in this case $r$ is exactly known and we compute the likelihood function over a wide range of parameter values. The panels of Fig. 1 show the likelihood function depicted against the parameters at different $k, k=1$ in panel (a), $k=5$ in (b) and $k=20$ in (c), respectively. The representation is in terms of contour plots (the lines are at $1 \%, 50 \%$, and $90 \%$ of the maximal value, the red cross indicates the values adopted to generate the measurements). After just one measurement, in (a), the likelihood function is symmetrical around a straight line, meaning ambiguity among infinite pairs of values. The following measurements bring relevant information and even at $k=5$ the likelihood function is an oval centered on a section of the previous line. After 20 measurements most ambiguity has vanished and the accurate estimation of the parameters is possible by, i.e., maximizing the likelihood function. The fact that the maximum of the likelihood function does not coincide exactly with the values used in the RSS generation is a consequence of the RSS noise and represents the estimation error.

\section{BAYESIAN FILTER AND RBPF IMPLEMENTATION}

The user's state at the time instant $k=0,1, \ldots$ is composed of both its position $\boldsymbol{\theta}_{k} \in \mathcal{A} \subset \mathcal{R}^{2}$ and its velocity

$$
\mathbf{x}_{k}=\left[\boldsymbol{\theta}_{k}^{T}, \dot{\boldsymbol{\theta}}_{k}^{T}\right]^{T}
$$

where $\mathcal{A}$ is the two dimensional indoor area under test and the coordinates are expressed according to a local Cartesian reference system. A number $N_{A P}$ of APs are deployed in known locations of $\mathcal{A}$, namely, the $\mathrm{j}$-th $\mathrm{AP}$ is in $\boldsymbol{\theta}_{A P, j}$. The RSS measurement $y_{j, k}$ from AP $j$ is drawn from a Gaussian pdf, conditioned on state and parameters. By explicating time dependence and user's state in (2) and (3) we find:

$$
y_{j, k}=h_{j}-10 \alpha_{j} \log _{10}\left(\left\|\boldsymbol{\theta}_{k}-\boldsymbol{\theta}_{A P, j}\right\| / d_{0}\right)+n_{j, k},
$$

where $h_{j}$ and $\alpha_{j}$ are the $\mathrm{j}$-th AP parameters and $n_{k}$ is a white zero-mean Gaussian process

$$
n_{j, k} \sim \mathcal{N}\left(0, \sigma_{y}^{2}\right) .
$$

Finally, independence is assumed among measurements of different APs, given the user's state. Our aim is to estimate the user's state $\mathbf{x}_{k}$ and the path-loss parameters $h_{j}$ and $\alpha_{j}$ for all APs based on the set of RSS measurements $\left\{y_{j, k}\right\}$.

\section{A. Bayesian filter}

The Bayesian filter computes the posterior pdf of user's state and path-loss parameters: ${ }^{1}$

$$
p\left(\mathbf{x}_{0: k},\left\{h_{j}, \alpha_{j}\right\}_{j=1: N_{A P}} \mid\left\{y_{j, 1: k}\right\}_{j=1: N_{A P}}\right),
$$

starting from suitable independent prior distributions that we will indicate, with a little abuse of notation, with $p_{0}(\mathbf{x})$ and $p_{0}\left(h_{j}, \alpha_{j}\right)$, respectively. The posterior pdf (7) can be decomposed in order to split the parameter estimation terms from the localization term:

$$
\begin{gathered}
p\left(\mathbf{x}_{0: k},\left\{h_{j}, \alpha_{j}\right\}_{j=1, \ldots, N_{A P}} \mid\left\{y_{j, 1: k}\right\}_{j=1, \ldots, N_{A P}}\right)= \\
\left(\prod_{j=1}^{N_{A P}} p\left(h_{j}, \alpha_{j} \mid \mathbf{x}_{0: k}, y_{j, 1: k}\right)\right) \cdot p\left(\mathbf{x}_{0: k} \mid\left\{y_{j, 1: k}\right\}_{j=1, \ldots, N_{A P}}\right) .
\end{gathered}
$$

Let us focus on the localization term, the last on the right of (8). A further factorization yields the recursive formulation

$$
\begin{aligned}
p\left(\mathbf{x}_{0: k} \mid\left\{y_{j, 1: k}\right\}_{j=1: N_{A P}}\right) & =\left(\prod_{j=1}^{N_{A P}} p\left(y_{j, k} \mid \mathbf{x}_{0: k}, y_{j, 1: k-1}\right)\right) \\
& \cdot p\left(\mathbf{x}_{k} \mid \mathbf{x}_{0: k-1},\left\{y_{j, 1: k-1}\right\}_{j=1: N_{A P}}\right) \\
& \cdot p\left(\mathbf{x}_{0: k-1} \mid\left\{y_{j, 1: k-1}\right\}_{j=1: N_{A P}}\right)
\end{aligned}
$$

The first term on the right hand side of (9) is the product of measurement likelihood functions, one per AP, which are conditionally independent. Each factor requires a marginalization over the path-loss parameters of the same AP, i.e., for the j-th factor:

$$
\begin{array}{r}
p\left(y_{j, k} \mid \mathbf{x}_{0: k}, y_{j, 1: k-1}\right)=\int_{h_{j}, \alpha_{j}} p\left(y_{j, k} \mid h_{j}, \alpha_{j}, \mathbf{x}_{0: k}, y_{j, 1: k-1}\right) \\
\cdot p\left(h_{j}, \alpha_{j} \mid \mathbf{x}_{0: k-1}, y_{j, 1: k-1}\right) \mathrm{d} h_{j} \mathrm{~d} \alpha_{j}
\end{array}
$$

where the likelihood function conditioned on the path-loss parameters, based on independence assumptions, results in

$$
p\left(y_{j, k} \mid h_{j}, \alpha_{j}, \mathbf{x}_{0: k}, y_{j, 1: k-1}\right)=p\left(y_{j, k} \mid h_{j}, \alpha_{j}, \mathbf{x}_{k}\right),
$$

and is given by (3).

\footnotetext{
${ }^{1}$ The notation $j=a: b$ stays for $j=a, a+1, \ldots, b$ and is used across the paper for shortness.
} 


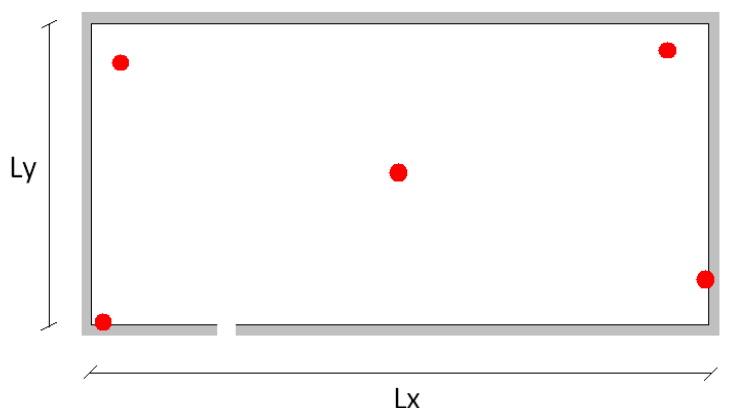

Fig. 2. Testbed adopted in the simulations; in the figure $L_{x}=40 \mathrm{~m}$ and $L_{y}=20 \mathrm{~m}$ and the APs are in the positions denoted by red circles.

The second term on the right hand side of (9) is the user's movement model; by assuming the Markov property, we simplify it in

$$
p\left(\mathbf{x}_{k} \mid \mathbf{x}_{0: k-1},\left\{y_{j, 1: k-1}\right\}_{j=1: N_{A P}}\right)=p\left(\mathbf{x}_{k} \mid \mathbf{x}_{k-1}\right) .
$$

The user's movement model depends on the type of user, e.g., a pedestrian or a robot, and two examples will be provided when discussing the results.

As for the parameter pdf, the update formula is obtained by means of the Bayes theorem, i.e., for the $\mathrm{j}$-th AP:

$$
\begin{aligned}
& p\left(h_{j}, \alpha_{j} \mid \mathbf{x}_{0: k}, y_{j, 1: k}\right)= \\
& \frac{p\left(y_{j, k} \mid h_{j}, \alpha_{j}, \mathbf{x}_{k}\right) \cdot p\left(h_{j}, \alpha_{j} \mid \mathbf{x}_{0: k}, y_{j, 1: k-1}\right)}{p\left(y_{j, k} \mid \mathbf{x}_{k}, y_{j, 1: k-1}\right)}
\end{aligned}
$$

where simplification (11) is employed and

$$
p\left(h_{j}, \alpha_{j} \mid \mathbf{x}_{0: k}, y_{j, 1: k-1}\right)=p\left(h_{j}, \alpha_{j} \mid \mathbf{x}_{0: k-1}, y_{j, 1: k-1}\right) .
$$

\section{B. Path-loss parameter model}

The model of the path-loss parameters should represent a fair compromise between accuracy and mathematical tractability. Our choice is to discretize the variables by defining a finite set of $N_{S}$ hypotheses for each AP, e.g., for the j-th AP:

$$
H_{j, s}=\left\{h_{j}^{s}, \alpha_{j}^{s}\right\}, s=1, \ldots, N_{S} .
$$

The values of the parameters can be sampled on either a suitable grid or according to some prior information. Their pdf is therefore represented by the set of probabilities of each hypothesis, whose update is found by means of the Bayes theorem, as in (13):

$$
\begin{aligned}
& \operatorname{Pr}\left(H_{j, s} \mid \mathbf{x}_{0: k}, y_{j, 1: k}\right)= \\
& \frac{p\left(y_{j, k} \mid H_{j, s}, \mathbf{x}_{k}\right) \cdot p\left(H_{j, s} \mid \mathbf{x}_{0: k-1}, y_{j, 1: k-1}\right)}{p\left(y_{j, k} \mid \mathbf{x}_{k}, y_{j, 1: k-1}\right)},
\end{aligned}
$$

for all $j$ and $s$. Finally, the integral in (10) results in the finite sum

$$
\begin{aligned}
& p\left(y_{j, k} \mid \mathbf{x}_{0: k}, y_{j, 1: k-1}\right)= \\
& \sum_{s=1}^{N_{S}} p\left(y_{j, k} \mid H_{j, s}, \mathbf{x}_{0: k}, y_{j, 1: k-1}\right) \cdot \operatorname{Pr}\left\{H_{j, s} \mid \mathbf{x}_{0: k-1}, y_{j, 1: k-1}\right\} .
\end{aligned}
$$

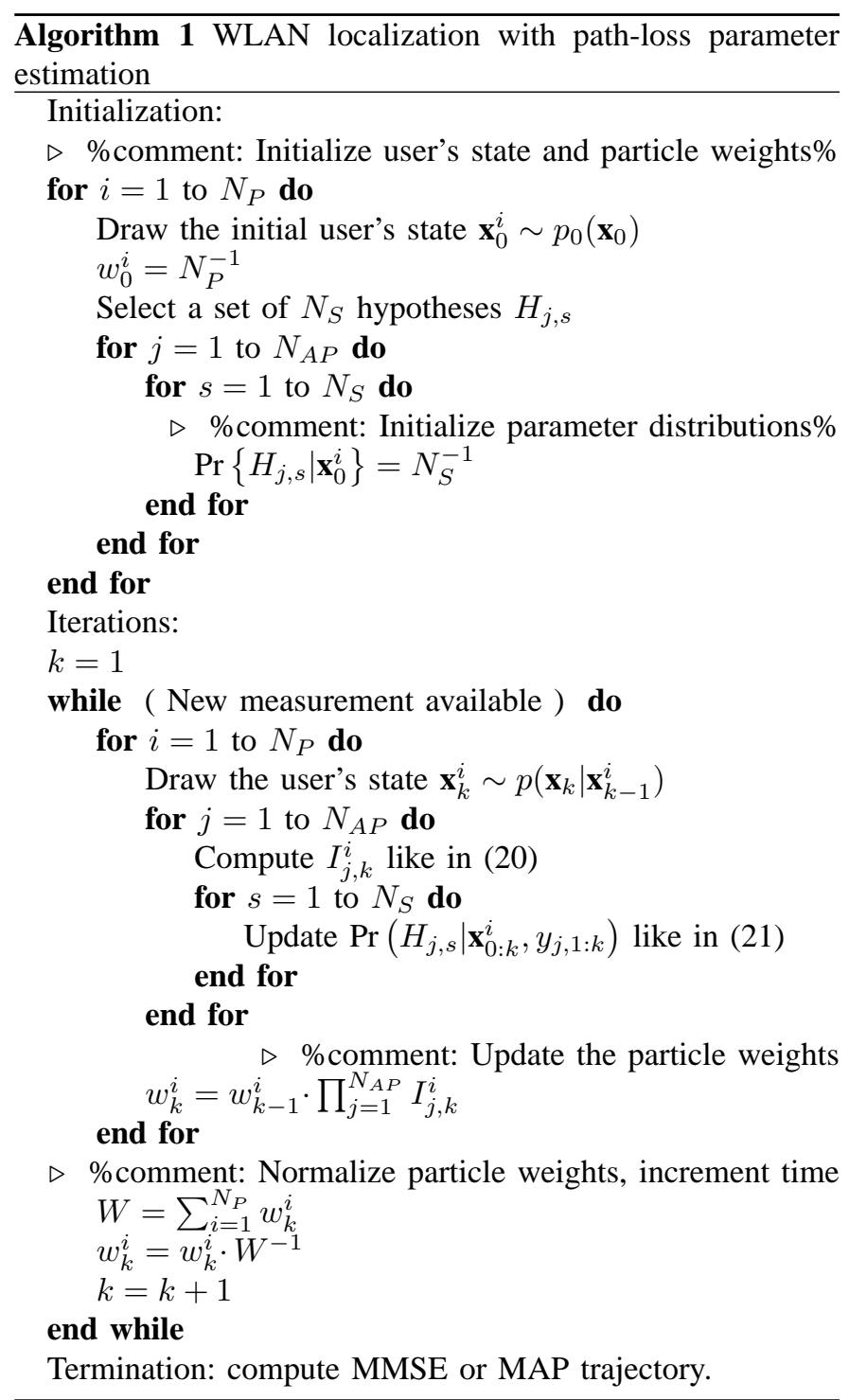

\section{RBPF implementation}

The Bayesian filter described in the previous Sections has been implemented by means of the RBPF [20] and is summarized in the algorithm box. Initialization is done by sampling the user's state from the prior pdf $p_{0}\left(\mathbf{x}_{0}\right)$ and by setting all the hypothesis probabilities to $N_{S}^{-1}$ for all $N_{P}$ particles. Then, for the i-th particle at the time instant $k>0$, the user's state is drawn according to

$$
\mathbf{x}_{k}^{i} \sim p\left(\mathbf{x}_{k} \mid \mathbf{x}_{k-1}^{i}\right)
$$

and the weight is computed from the RSS likelihood function

$$
w_{k}^{i}=w_{k-1}^{i} \cdot\left(\prod_{j=1}^{N_{A P}} p\left(y_{j, k} \mid \mathbf{x}_{0: k}^{i}, y_{j, 1: k-1}\right)\right),
$$




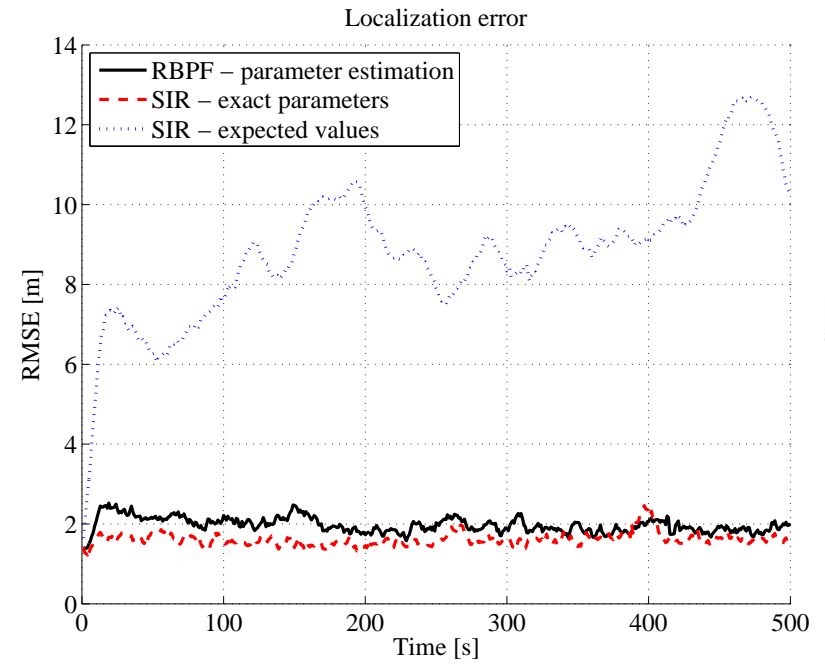

(a) RMSE

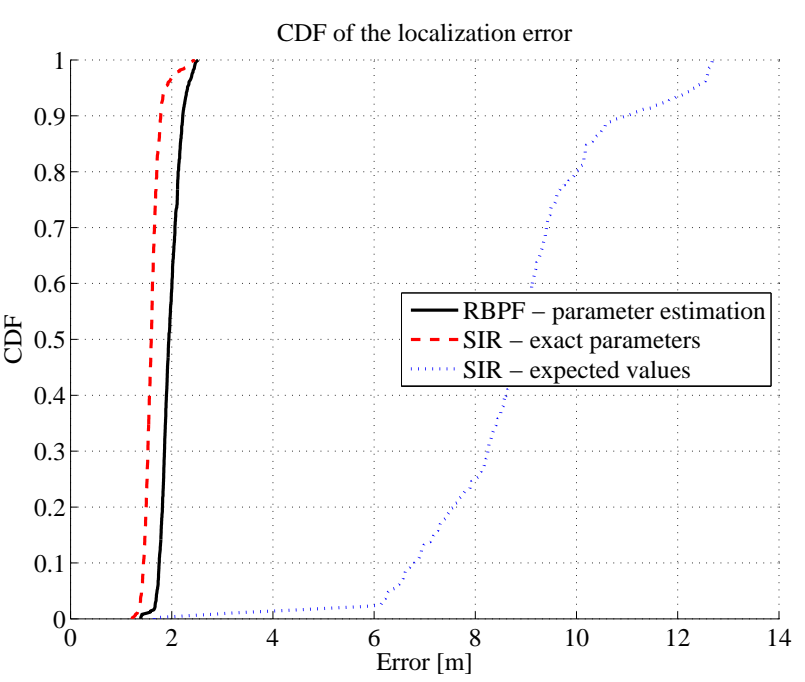

(b) $\mathrm{CDF}$

Fig. 3. Simulation results in the testbed of Fig. 2 in terms of the RMSE of the proposed algorithm (black continuous curve), SIR fed with true parameters (red dashed) and SIR with expected parameters (blue dotted); the simulations were run with 1000 particles and RSS noise variance $\sigma_{y}^{2}=5$ and the results were averaged over 100 independent realization; we propose (a) the RMSE against time, (b) the empirical CDF of the RMSE.

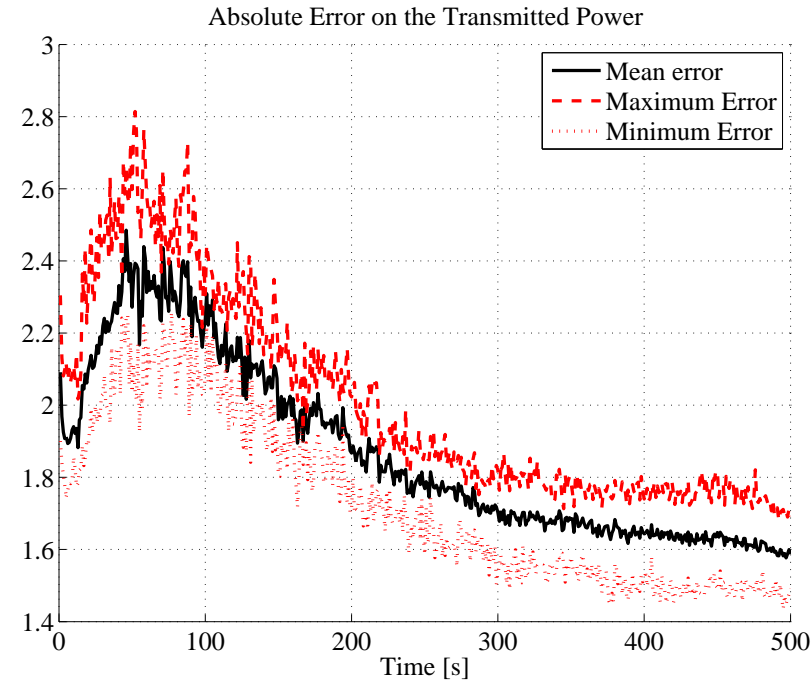

(a) Transmitted Power

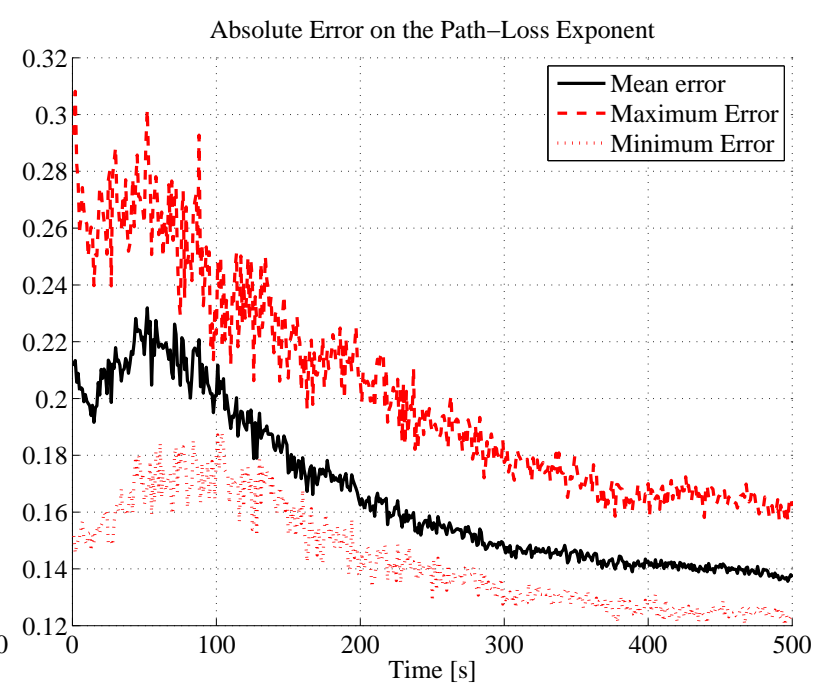

(b) Pah-Loss Exponent

Fig. 4. Estimation of parameters in simulation of Fig. 3: we depict the absolute error on (a) the transmitted power and (b) the path-loss exponent; for visualization issues we depict in all cases only the maximum, minimum and mean absolute error over the 5 APS.

in which the parameter pdf is involved like in (17)

$$
\begin{aligned}
& I_{j, k}^{i} \hat{=} p\left(y_{j, k} \mid \mathbf{x}_{0: k}^{i}, y_{j, 1: k-1}\right)= \\
& \sum_{s=1}^{N_{S}} p\left(y_{j, k} \mid H_{j, s}, \mathbf{x}_{0: k}^{i}, y_{j, 1: k-1}\right) \cdot \operatorname{Pr}\left\{H_{j, s} \mid \mathbf{x}_{0: k-1}^{i}, y_{j, 1: k-1}\right\} .
\end{aligned}
$$

As last step of the iteration, the hypothesis probabilities are updated according to (16), with

$$
\begin{aligned}
& \operatorname{Pr}\left(H_{j, s} \mid \mathbf{x}_{0: k}^{i}, y_{j, 1: k}\right)= \\
& \frac{p\left(y_{j, k} \mid H_{j, s}, \mathbf{x}_{k}^{i}\right) \cdot p\left(H_{j, s} \mid \mathbf{x}_{0: k-1}^{i}, y_{j, 1: k-1}\right)}{p\left(y_{j, k} \mid \mathbf{x}_{k}^{i}, y_{j, 1: k-1}\right)}
\end{aligned}
$$

for all hypotheses and APs. When the algorithm terminates, the estimated trajectory can be computed by either averaging over the particles to obtain the Minimum Mean Square error (MMSE) estimator or maximizing it (Maximum A-Posteriori - MAP - estimator).

The computational complexity required by the proposed algorithm is linearly proportional to the number of particles, $N_{P}$, to the number of APs, $N_{A P}$, and to the number of hypotheses, $N_{S}$. The advantage of applying RBPF is that the state sampling, that is the heaviest operation, refers only to the user's state, whose dimensionality does not depend on the number of APs and parameters. These latter are only involved 


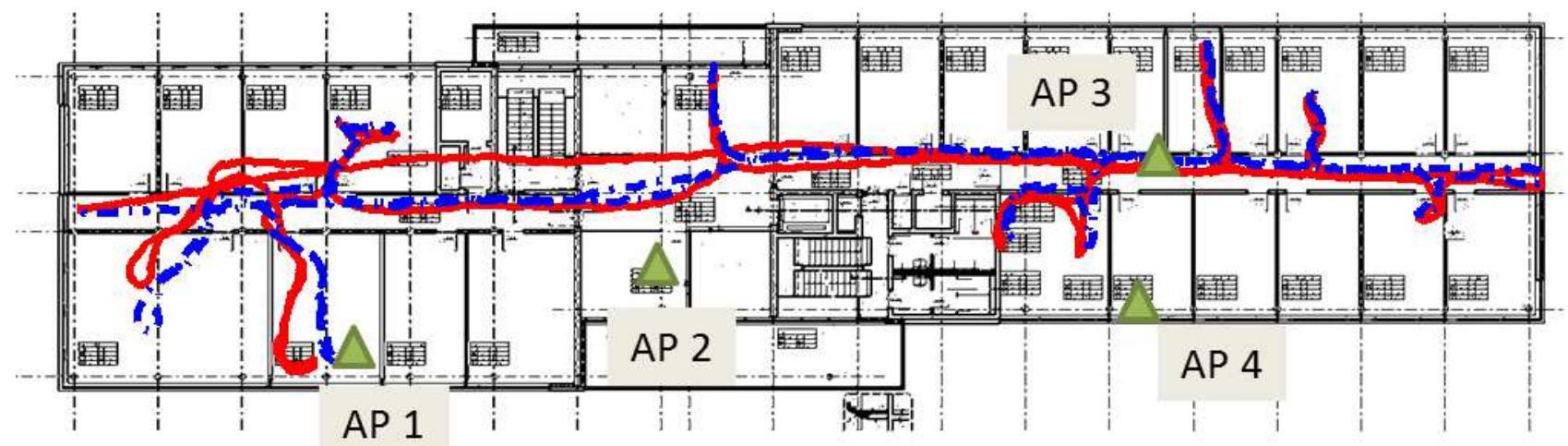

Fig. 5. Experiment in a fairly $65 \times 20 \mathrm{~m}$ office floor with 4 APs: the trajectory in dashed blue line is obtained by applying FootSLAM [19] and is here considered the ground truth, while the estimated trajectory is denoted by a red continuous line; the AP's true positions are denoted by green triangles and they are employed by the algorithm; the trajectories are arranged in the floor layout, that is not known by the algorithm. Here, 1000 particles and $\sigma_{y}=5 \mathrm{dBm}$ are employed.

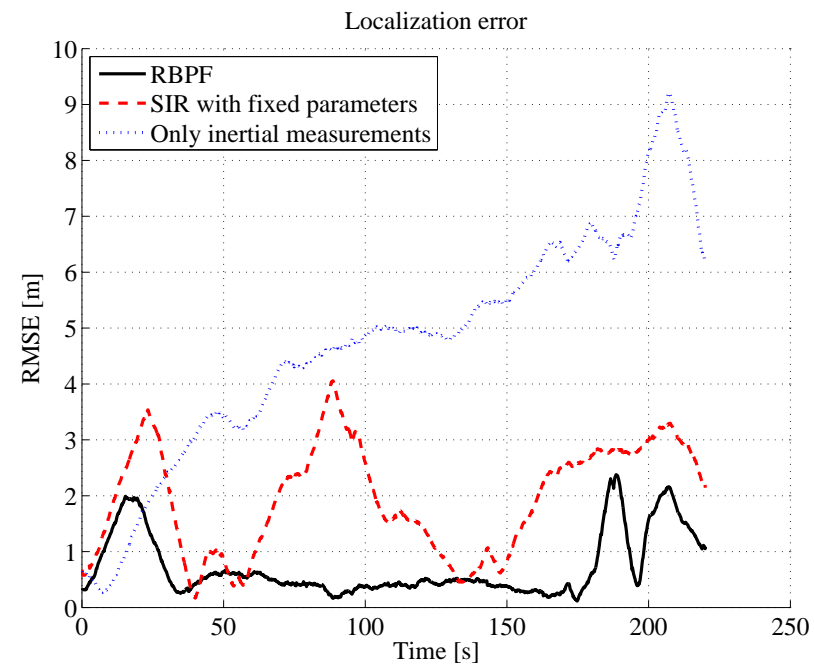

(a) RMSE

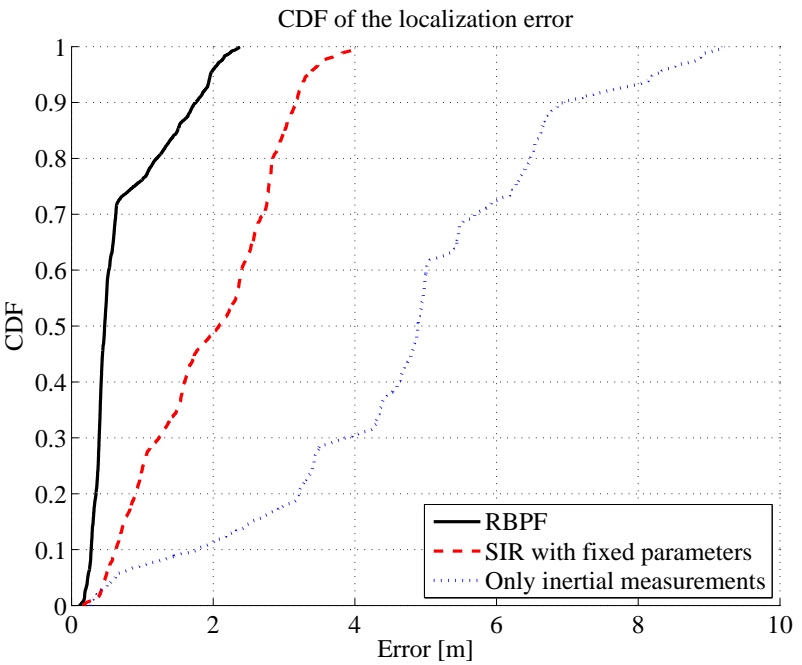

(b) $\mathrm{CDF}$

Fig. 6. Localization error in the experiment of Fig. 5, comparing our proposal (black continuous lines), SIR with fixed parameters (red dashed lines) and IMU's based localization (blue dotted lines); we propose (a) the localization error against time and (b) the empirical CDF.

in the evaluation of the conditional RSS likelihood functions, which can be efficiently done in logarithmic domain.

\section{Simulations}

We propose a preliminary validation of the algorithm by means of simulations. The synthetic testbed is depicted in Fig. 2 and is a $40 \times 20 \mathrm{~m}$ open area where 5 APs are denoted by red circles and they emit a beacon signal with a constant period $T$.

The user's state transition (18)

$$
p\left(\mathbf{x}_{k} \mid \mathbf{x}_{k-1}^{i}\right), k=1,2, \ldots,
$$

is described in this simulation by means of the popular Nearly Constant Velocity Model (NCVM) sampled at time instants $k T$ [21]

$$
\mathbf{x}_{k+1}=F \mathbf{x}_{k}+\mathbf{v}_{k}, k=0,1,2, \ldots,
$$

in which the state is, like before, the 4-dimensional vector composed by the user's position and velocity and $\mathbf{v}_{k}$ is a zero mean white Gaussian process. In (22) the $4 \times 4$ matrix $F$ is defined like

$$
F=\left(\begin{array}{ll}
1 & \tau \\
0 & 1
\end{array}\right) \otimes \mathbf{I}_{2},
$$

having for simplicity introduced the identity matrix $\mathbf{I}_{2}$ of order 
2 and the Kronecker product $\otimes$. The covariance matrix $Q$ of the noise $\mathbf{v}_{k}$ is

$$
Q=E\left[\mathbf{v}_{k} \mathbf{v}_{k}^{\prime}\right]=\sigma_{v}^{2}\left(\begin{array}{cc}
\frac{1}{3} \tau^{3} & \frac{1}{2} \tau^{2} \\
\frac{1}{2} \tau^{2} & \tau
\end{array}\right) \otimes \mathbf{I}_{2},
$$

where $\sigma_{v}^{2}$ is the noise variance and multiplies all entries. The initial state $\mathbf{x}_{0}$ is drawn from a Multivariate Gaussian (MG) prior distribution with diagonal covariance matrix; the standard deviations are set to $1 \mathrm{~m}$ for the position coordinates $\left(\sigma_{\theta_{1}}\right.$ and $\left.\sigma_{\theta_{2}}\right)$ and $0.1 \mathrm{~m} / \mathrm{s}$ for the velocity components $\left(\sigma_{\dot{\theta}_{1}}\right.$ and $\left.\sigma_{\dot{\theta}_{2}}\right)$. We draw the independent parameter values $h_{j}$ and $\alpha_{j}$ for each AP from two Gaussian distributions with known means, $h_{0}$ and $\alpha_{0}$, and diagonal covariance matrices; in all the simulations the standard deviation of the starting distributions are set to $\sigma_{h}=3 \mathrm{dBm}$ and $\sigma_{\alpha}=0.3$, respectively, and 1000 particles are employed.

The parameter hypotheses, $\left\{H_{s}\right\}, s=1: N_{S}$, are represented by all the couples $\left\{h_{j}^{s}, \alpha_{j}^{s}\right\}$ obtained by the combinations of the values $h_{j}^{s}$ from $-50 \mathrm{dBm}$ up to $-30 \mathrm{dBm}$ with step $1 \mathrm{dBm}$ and $\alpha$ from 1.5 up to 3.5 with step 0.1 .

The black curves in both panels of Fig. 3 refer to the localization error achieved in the simulation scenario of Fig. 2 . The RSS measurements have been generated with variance $\sigma_{y}^{2}=5$ and the walk lasts $K=500$ seconds at the pace of $T=1 \mathrm{~s}$ (that is 1 measurement per second). We compute the Minimum Mean Square Error (MMSE) estimator of the whole trajectory, whose MSE is averaged on $I=100$ independent realizations. For simplicity, we depict in our figures the root MSE (RMSE), since its dimensions are in meters; in detail, panel (a) shows the localization RMSE against time while panel (b) reports on its empirical cumulative density function (CDF).

In the same Fig. 3 we draw two other set of curves. The red dashed ones refer to performance of the algorithm with perfect knowledge of the parameters, i.e., only one hypothesis is considered, $N_{S}=1$, which corresponds to the values adopted in data generation. This case is equivalent to apply the Sampling Importance Resampling (SIR) algorithm where the state space is composed only of the user's state [22]. Also the blue dotted curves are obtained by assuming only one hypothesis, but the values here are the prior means $h_{0}$ and $\alpha_{0}$. In this latter case the mismatch on the parameters will be on the same order of magnitude as the prior pdf's standard deviations, $3 \mathrm{dBm}$ for $h$ and 0.3 for $\alpha$. Although such deviations are not that large, we can see in Fig. 3 how big the produced error can be, and in panel (a) we notice that the blue curve diverges. On the other hand our proposal is very close to the red curve, since the model mismatch is highly mitigated by the parameter estimation. This latter is explicitly reported in Fig. 4 for the same simulation: panel (a) refers to the transmitted power estimation and shows the average, maximum and minimum absolute error $\|\Delta h\|$, of the estimation within all $5 \mathrm{APs}^{2}$. The same is done for $\alpha$ in panel

\footnotetext{
${ }^{2}$ The mean of the parameter pdf is used in the computation of the absolute error.
}

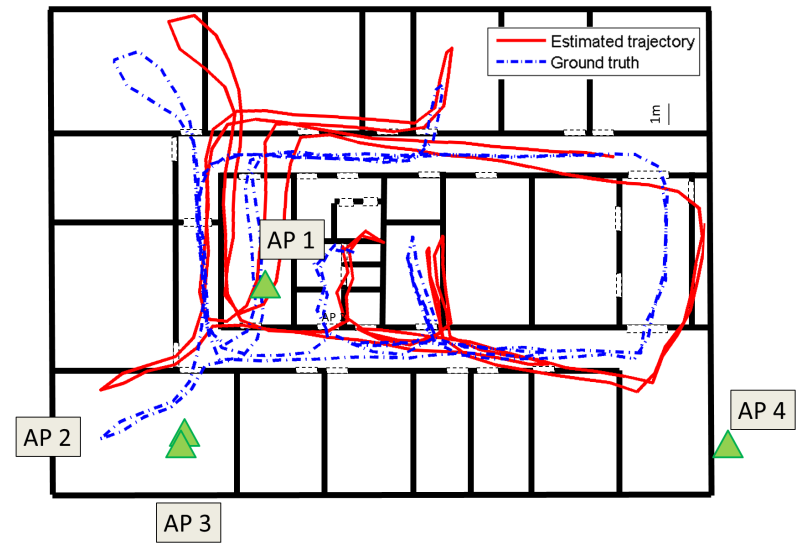

Fig. 7. Experiment in a $45 \times 25 \mathrm{~m}$ office floor with 4 APs; notation and experiment parameters are the same as in Fig. 5.

(b) and in both cases we notice that after an initial transient the absolute errors clearly decrease; at the end of the simulation the average absolute error is reduced by $60 \%-70 \%$ with respect to the maximum in both cases.

\section{EXPERIMENTS}

Two experiments have been carried out in different buildings and configurations in order to validate our algorithm. The user's state model (18) is here provided by the output of a foot-mounted IMU: the raw inertial measurements are filtered by a ZUPT algorithm and the resulting step measurements are then used to sample the new user's state [18], [19]. The RSS measurements are collected by means of either a laptop or a mobile phone. The use of an expensive and invasive foot mounted IMU is realistic in a professional application, like a Disaster Management scenario, but not for commercial massmarket services; in the latter case it can be replaced by the inertial sensor available in most smartphones: their inaccuracy will be a challenge to afford in the close future.

The first experiment takes place in an office floor which is about $65 \mathrm{~m}$ long and $20 \mathrm{~m}$ wide, where the user walks for about 3 minutes back and forth the hallways and some rooms. The user equipment is composed of a foot mounted IMU and a hand-held smartphone which logs the RSS measurements from 4 APs, within a IEEE 802.11 (WiFi) b/g network; the processing has been done off-line. We notice that RSS measurements from different APs are usually not synchronous and this is accounted for in the algorithm by assigning dummy values to the weights $I_{j, k}^{i}$ of (20), for all $i$, which refer to missing measurements. The parameter hypotheses, $\left\{H_{s}\right\}, s=1: N_{S}$, are obtained by the combinations of the values $h_{j}^{s}$ from $46 \mathrm{dBm}$ up to $-30 \mathrm{dBm}$ with step $2 \mathrm{dBm}$ and $\alpha$ from 1 up to 4 with step 0.5 . The standard deviation of the RSS measurements is set to $\sigma_{y}=5 \mathrm{dBm}$.

Fig. 5 shows the layout of the testbed with the AP's locations (manually evaluated) and two trajectories: in blue (dashed line) the ground-truth and in red (continuous line) the trajectory estimated by our proposal. The ground-truth 


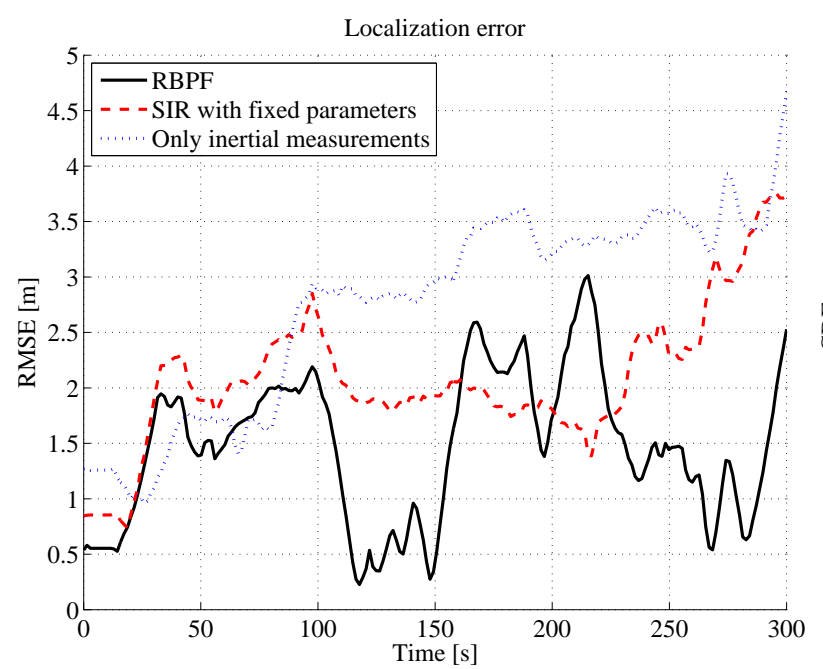

(a) RMSE

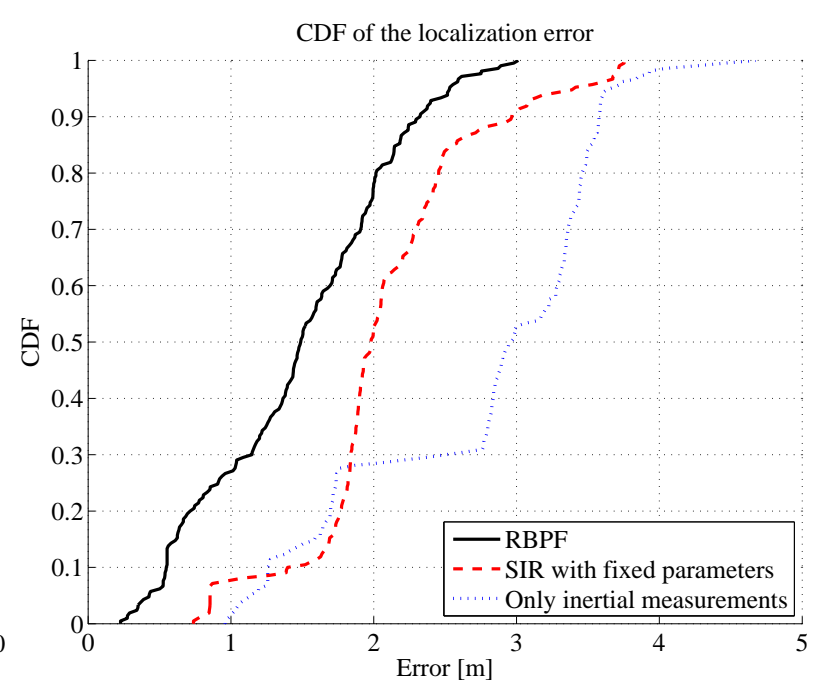

(b) $\mathrm{CDF}$

Fig. 8. Localization error in the experiment of Fig. 7, comparing our proposal (black continuous lines), SIR with fixed parameters (red dashed lines) and IMU's based localization (blue dotted lines); we propose (a) the localization error against time and (b) the empirical CDF.

has been evaluated by means of FootSLAM, which is a Simultaneous Localization And Mapping (SLAM) algorithm based on the IMU only and presented in [19]; FootSLAM, in this case run with as many as $5 \times 10^{4}$ particles, provides trajectories within a sub-meter error, as the matching with the map layout confirms. From a first inspection of the results we can point out two issues of interest. On the right side of the floor the estimation is very accurate, both in the hallway and in the rooms, due to the proximity of the APs to the trajectory, especially AP 3. On the left side of the floor, instead, localization suffers from the disposition of the APs and the error increases. The black continuous curves in Fig. 6 quantify the localization error - against time in panel (a) and the corresponding CDF in panel (b). We can see that at the far ends of the walk, corresponding to the user walking in the left part of the floor, the error grows up to 2 meters, while it is much below 1 meter in the other case. In the same figure we also depict the comparison with the result of two other algorithms: the red dashed lines refer to the SIR algorithm fed with the average parameter values, $\alpha=2$ and $h=-40 \mathrm{dBm}$, while the blue dotted curves refer to the algorithm which does not make use of RSS measurements, but only of the IMU's measurements.

The algorithm with fixed parameters has a very unstable behavior, since the localization error alternates low values to 4-meter-peaks, due to model mismatches. Changing the parameter values yields a different disposition of the error peaks but does not improve the algorithm stability. Furthermore, using only IMU's step measurements brings to a drift in the localization error, as widely documented in the literature [18], [19] and, in our case, the error amounts already to 6-7 meters after 3 minutes. Nonetheless, using IMU to propose the user's state has a strong impact in mitigating inaccuracy if the user walks a short time in a part of the floor that is not well covered by the APs.

Fig. 7 presents the second experiment, performed in another office environment about $45 \mathrm{~m}$ long and $25 \mathrm{~m}$ wide, with a square hallway and 4 APs. The user walks about 7 minutes - corresponding to 3 rounds in the hallway with visits to some of the offices. In this case the RSS are collected by a hand-held laptop while the foot-mounted IMU is still used to obtain step measurements. In this scenario the results are worse than in the first experiment, due especially to a little rotation in the estimated trajectory (red curve in Fig. 7). We notice, however, that this setting is harder since, although 4 APs are still deployed, two APs - AP 2 and 3 - are located in the same position, thus reducing the area coverage and, above all, signal diversity. However, the localization error depicted in Fig. 8 highlights a performance gain with respect to the algorithm with fixed parameters - the error is below 2 meters for $80 \%$ of time.

\section{CONCLUSION}

We proposed a localization algorithm for indoor environments based on RSS measurements, which are modeled by means of the path-loss model. The algorithm was developed in the framework of Bayesian probability theory and accounts for the path-loss model calibration. In detail, we dealt with the transmitted power and the path-loss exponent, that are usually not known in real scenarios, at least with accuracy. Our algorithm is able to gradually estimate such parameters together with the user's trajectory, without any previous calibration phase.

The theoretical Bayesian filter is implemented by means of a Rao-Blackwellized particle filter, where a state transition model is assumed in order to propose the new user's position and the RSS measurements are, then, used to weight the particles. The path-loss parameters are defined in terms of a 
probability distribution that is updated after each measurement. This representation has two main benefits: only the user's state is sampled, so the complexity of this operation is not related to the presence of parameters, and, above all, the parameter estimation is not point wise but it is gradually improved, related to the quantity of information available. This avoids over-estimation issues and makes the algorithm robust.

Validation of the proposed algorithm has been carried out by means of both simulations and experiments. For the latter, two different buildings were employed and fusion with inertial data derived from a foot-mounted IMU was also discussed.

Future challenges follow mainly two directions. The former is represented by a better characterization of radio propagation, above all in near field conditions, where the traditional path-loss model turns out to be increasingly inaccurate. The latter challenge is instead represented by three dimensional scenarios, which are not mere extensions of two dimensions, but offer different issues.

\section{REFERENCES}

[1] W. Kolodziej and J. Hjelm. Local positioning systems: LBS applications and services. CRC Press, 2006.

[2] S.A. Ahson and M. Mohammad Ilyas. Location-Based Services Handbook: Applications, Technologies, and Security. CRC Press, 2009.

[3] P. Misra and P. Enge. Global Positioning System, Signals, Measurements, and Performance. Ganga-Jamuna Press, 2006.

[4] J. Rantakokko, J. Rydell, P. Stromback, P. Handel, J. Callmer, D. Tornqvist, F. Gustafsson, and M. Grudén. Accurate and reliable soldier and first responder indoor positioning: multisensor systems and cooperative localization. IEEE Wireless Commun., 18(2), pages 10-18, 2011.

[5] A.H. Sayed, A. Tarighat, and N. Khajehnouri. Network-based wireless location: challenges faced in developing techniques for accurate wireless location information. IEEE Signal Processing Mag., 22(4), pages 24-40, 2005.

[6] Thorsten Vaupel, Jochen Seitz, Frederic Kiefer, Stephan Haimerl, and Jorn Thielecke. Wi-fi positioning: System considerations and device calibration. In Indoor Positioning and Indoor Navigation (IPIN), 2010 International Conference on, pages 1 -7, 2010.

[7] P. Bahl and V. Padmanabhan. RADAR: An in-building RF-based user location and tracking system. Proc. IEEE INFOCOM, pages 775-784, Mar 2000.

[8] A. Haeberlen, E. Flannery, A. Ladd, A. Rudys, D. Wallace, and L. Kavraki. Practical robust localization over large-scale 802.11 wireless networks. In Proceedings of MobiCom 04, ACM, pages 70-84, Oct 2004.

[9] Jie Yin, Qiang Yang, and Lionel M. Ni. Learning adaptive temporal radio maps for signal-strength-based location estimation. IEEE Transactions on Mobile Computing, 7, pages 869-883, 2008.

[10] S. Fang and C. Wang. A dynamic hybrid projection approach for improved Wi-Fi location fingerprinting. Vehicular Technology, IEEE Transactions on, 60(3), pages 1037-1044, 2011.

[11] J. Parsons. The Mobile Radio Propagation Channel, $2^{n} d$ Ed. Wiley \& Sons, November 2000.

[12] L. Bruno P. Addesso and R. Restaino. Integrating RSS from unknown access points in WLAN positioning. In Wireless Networking Symposium (IWCMC2011-Wireless Nets), Istanbul, Turkey, 72011.

[13] Paolo Addesso, Luigi Bruno, and Rocco Restaino. Adaptive localization techniques in $\mathrm{WiFi}$ environments. In Wireless Pervasive Computing (ISWPC), 2010 5th IEEE International Symposium on, pages 289 -294, May 2010.

[14] A. Bose and Chuan Heng Foh. A practical path loss model for indoor WiFi positioning enhancement. In Information, Communications Signal Processing, 2007 6th International Conference on, pages 1-5, 2007.

[15] Xinrong Li. RSS-based location estimation with unknown pathloss model. Wireless Communications, IEEE Transactions on, 5(12), pages 3626-3633, 2006
[16] Qing Zhang, Chuan Heng Foh, Boon-Chong Seet, and A. C. Fong. Variable elasticity spring-relaxation: improving the accuracy of localization for WSNs with unknown path loss exponent. Personal Ubiquitous Comput., 16(7), pages 929-941, October 2012.

[17] H. Nurminen, J. Talvitie, S. Ali-Loytty, P. Muller, E. Lohan, R. Piche, and M. Renfors. Statistical path loss parameter estimation and positioning using RSS measurements in indoor wireless networks. In Indoor Positioning and Indoor Navigation (IPIN), 2012 International Conference on, pages 1-9, 2012.

[18] E. Foxlin. Pedestrian tracking with shoe-mounted inertial sensors. IEEE Comput. Graph. Appl., 25(6), pages 38-46, 2005.

[19] M. Angermann and P. Robertson. FootSLAM: Pedestrian simultaneous localization and mapping without exteroceptive sensors - hitchhiking on human perception and cognition. Proceedings of the IEEE, 100(Special Centennial Issue), pages $1840-1848,132012$.

[20] B. Ristic, S. Arulampalam, and N. Gordon. Beyond the Kalman filter: particle filters for tracking applications. Artech House Publishers, 2004.

[21] Y. Bar-Shalom, X.R. Li, and T. Kirubarajan. Estimation with Applications to Tracking and Navigation: Theory Algorithms and Software. John Wiley And Sons, 2001.

[22] M.S. Arulampalam, S. Maskell, N. Gordon, and T. Clapp. A tutorial on particle filters for online nonlinear/non-Gaussian Bayesian tracking. IEEE Trans. On Signal Processing., 50(2), pages 174-188, Feb 2002. 